\title{
Thrombus Aspiration in ThrOmbus containing culpRiT lesions in Non-ST-Elevation Myocardial Infarction (TATORT-NSTEMI): study protocol for a randomized controlled trial
}

Suzanne de Waha', Ingo Eitel', Steffen Desch¹, Bruno Scheller², Michael Böhm², Bernward Lauer ${ }^{3}$, Meinrad Gawaz ${ }^{4}$, Tobias Geisler ${ }^{4}$, Oliver Gunkel ${ }^{5}$, Leonhard Bruch ${ }^{6}$, Norbert Klein $^{7}$, Dietrich Pfeiffer ${ }^{7}$, Gerhard Schuler$^{1}$, Uwe Zeymer ${ }^{8 \dagger}$ and Holger Thiele ${ }^{1 * \dagger}$

\begin{abstract}
Background: Current guidelines recommend thrombus aspiration in patients with ST-elevation myocardial infarction (STEMI); however, there are insufficient data to unequivocally support thrombectomy in patients with non-STEMI (NSTEMI).

Methods/Design: The TATORT-NSTEMI (Thrombus Aspiration in ThrOmbus containing culpRiT lesions in Non-ST -Elevation Myocardial Infarction) trial is a prospective, controlled, multicenter, randomized, open-label trial enrolling 460 patients. The hypothesis is that, against a background of early revascularization, adjunctive thrombectomy leads to less microvascular obstruction (MO) compared with conventional percutaneous coronary intervention (PCI) alone, as assessed by cardiac magnetic resonance imaging (CMR) in patients with NSTEMI. Patients will be randomized in a 1:1 fashion to one of the two treatment arms. The primary endpoint is the extent of late MO assessed by CMR. Secondary endpoints include early $\mathrm{MO}$, infarct size, and myocardial salvage assessed by CMR as well as enzymatic infarct size and angiographic parameters, such as thrombolysis in myocardial infarction flow post- $\mathrm{PCl}$ and myocardial blush grade. Furthermore, clinical endpoints including death, myocardial re-infarction, target vessel revascularization, and new congestive heart failure will be recorded at 6 and 12 months. Safety will be assessed by the incidence of bleeding and stroke.
\end{abstract}

Summary: The TATORT-NSTEMI trial has been designed to test the hypothesis that thrombectomy will improve myocardial perfusion in patients with NSTEMI and relevant thrombus burden in the culprit vessel reperfused by early $\mathrm{PCl}$.

Trial registration: The trial is registered under www.clinicaltrials.gov: NCT01612312.

Keywords: Non-ST-elevation myocardial infarction, Thrombectomy, Cardiac magnetic resonance imaging, Microvascular obstruction

\footnotetext{
*Correspondence: thielh@medizin.uni-leipzig.de

${ }^{\dagger}$ Equal contributors

'Department of Internal Medicine/Cardiology, University of Leipzig - Heart

Center, Struempellstr. 39, Leipzig 04289, Germany

Full list of author information is available at the end of the article
} 


\section{Background}

Use of manual thrombectomy devices during percutaneous coronary intervention (PCI) in patients with acute ST-elevation myocardial infarction (STEMI) has been shown to decrease the incidence of cardiac death and repeat myocardial infarction [1-3]. Consequently, current guidelines of the European Society of Cardiology (ESC) and the American Heart Association/American College of Cardiology (AHA/ACC) recommend manual thrombectomy in patients with STEMI (class IIa, level of evidence A, and class IIa, level of evidence B, respectively) $[4,5]$. However, approximately 50 to $70 \%$ of all patients with non-ST-elevation myocardial infarction (NSTEMI) also display a relevant thrombus burden in the culprit vessel $[6,7]$. Furthermore, with rates of angiographic noreflow phenomenon ranging from 15 to $40 \%$, depending on thrombus burden and intra-procedural thrombotic events, only suboptimal reperfusion success can be achieved in a significant portion of patients with NSTEMI [8-10]. Thus, thrombectomy in patients with NSTEMI may represent a useful intervention, with the potential to improve patient prognosis. However, current guidelines do not give a clear recommendation for thrombus aspiration in NSTEMI [11,12]. This is mainly because of the lack of data on thrombus aspiration in NSTEMI, as only one small prospective observational study has been published to date [7]; in that 70-patient study, thrombectomy had a similar effectiveness rate to that reported in patients with STEMI.

This emphasizes the need for an adequately powered randomized controlled trial (RCT) to address the current role of thrombus aspiration in patients with NSTEMI and a relevant thrombus burden in the culprit vessel. The TATORT-NSTEMI (Thrombus Aspiration in ThrOmbus containing culpRiT lesions in Non-ST-Elevation Myocardial Infarction) trial is designed to test the hypothesis that against a background of early revascularization, adjunctive thrombectomy leads to less microvascular obstruction (MO) compared with conventional PCI alone, as assessed by cardiac magnetic resonance imaging (CMR).

\section{Methods/design}

The TATORT-NSTEMI trial is a prospective, multicenter, randomized, controlled, open-label study to compare PCI plus routine thrombus aspiration with PCI without thrombus aspiration in patients with NSTEMI and relevant thrombus burden who are undergoing early invasive reperfusion therapy. The study will determine if adjunctive thrombectomy is superior than PCI alone with respect to the extent of no-reflow/MO assessed by CMR in patients with NSTEMI.

\section{Primary and secondary endpoints}

The primary study endpoint of the TATORT-NSTEMI trial is the extent of late MO assessed by CMR on days 1 to 4 after randomization. Using CMR, zones of $\mathrm{MO}$ within the infarcted myocardium can be visualized and quantified, representing the CMR equivalent of the noreflow phenomenon $[13,14]$. MO assessed by CMR has not only been shown to be associated with adverse functional outcome after myocardial infarction $[15,16]$, but also with adverse clinical outcome including mortality [17-19]. Further, late $\mathrm{MO}$ assessed 15 minutes after contrast-medium injection has been shown to have higher prognostic impact than early MO assessed 1 to 2 minutes after gadolinium administration or by first-pass gadolinium-enhanced perfusion $[17,20]$. The mechanistic hypothesis of the current trial is that thrombus aspiration will result in reduction of distal embolization, thus preserving microvascular integrity, reflected by less late MO, which will translate into improved clinical outcome.

Secondary endpoints include other CMR parameters associated with patient prognosis, such as early MO, infarct size, myocardial salvage, and left ventricular ejection fraction (LVEF), as well as angiographic markers of reperfusion success such as the thrombolysis in myocardial infarction (TIMI) flow post-PCI and myocardial blush grade $[18,21]$. In addition, troponin $\mathrm{T}$ after 24 and 48 hours for determination of enzymatic infarct size will be evaluated. Further, a combined clinical endpoint including death, re-infarction, target vessel revascularization, and congestive heart failure (CHF) will be assessed after 6 and 12 months. Death is defined as death from any cause, but will be divided into cardiac and noncardiac causes, and will be regarded as cardiac in origin unless obvious non-cardiac causes can be identified. The diagnosis of re-infarction during the index hospitalization will be based on clinical symptoms, new ST-segment changes, and an increase in the creatine kinase-MB levels above the reference limits in patients with normalized values, or if there is an increase of more than $20 \%$ from the most recent non-normalized measurement. At follow-up, any new ischemic symptoms leading to hospital admission accompanied by raised troponin levels will be defined as re-infarction [22]. New CHF after randomization during hospital stay will be defined as $\mathrm{CHF}$ when there is at least one of the following conditions and it requires treatment with diuretics (cardiogenic shock, pulmonary edema, or signs of pulmonary congestion on X-ray, rales of greater than one-third from lung basis, pulmonary capillary wedge pressure $>25 \mathrm{mmHg}$, or dyspnea with blood oxygen saturation $<90 \%$ in the absence of lung disease). After hospital discharge, any new CHF leading to re-hospitalization will be counted. 
Clinical outcome will be assessed by a telephone interview at 6 and 12 months. Any clinical event will be verified by hospital or general practitioner records. Finally, assessment of quality of life will be performed using the EuroQol-5D (www.euroqol.org) questionnaire during the 6-month and 12-month follow-ups.

Safety assessment will include stroke and bleeding until hospital discharge. Stroke will be defined as any new neurological symptoms in association with signs of ischemia or hemorrhage in cranial computed tomography or magnetic resonance imaging. Bleeding will be defined in accordance with the GUSTO (Global Utilization of Streptokinase and t-PA for Occluded Coronary Arteries) criteria as 1) severe or life-threatening, 2) moderate, or 3) mild bleeding [23]. However, based on previous studies, thrombus aspiration is associated with only modest complications, therefore no major safety concern for thrombus aspiration is expected. All

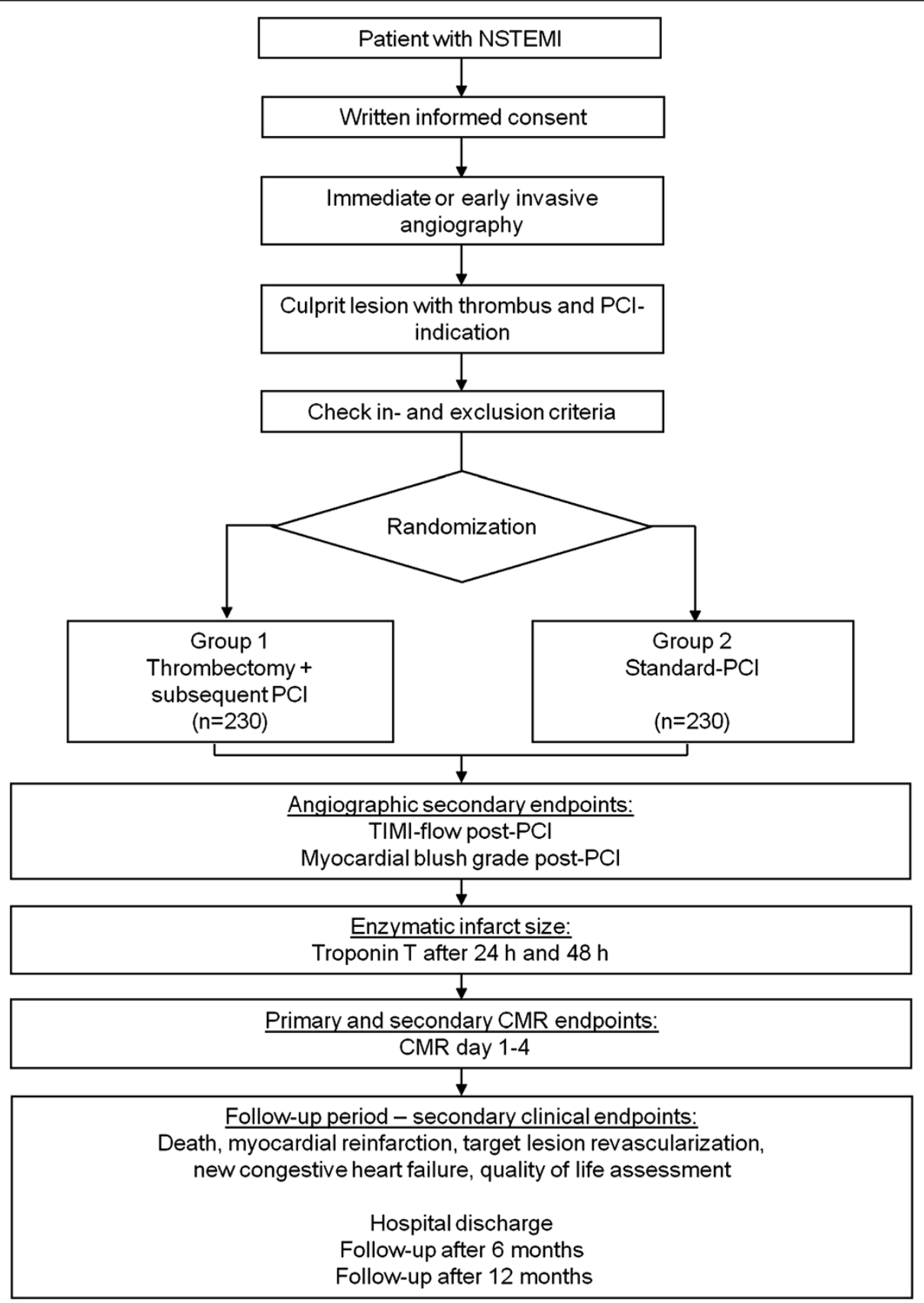

Figure 1 Study flow chart. CMR, cardiac magnetic resonance; NSTEMI, non-ST-elevation myocardial infarction; PCI, percutaneous coronary intervention; TIMI, thrombolysis in myocardial infarction. 
safety aspects will be monitored by a Data Safety Monitoring Board (DSMB). All clinical and safety endpoints will be adjudicated by a Clinical Endpoints Committee (CEC) blinded to treatment assignment, based on data provided by the clinical-trial sites.

\section{Patient population}

The study population will consist of 460 patients with NSTEMI enrolled at eight centers in Germany. Informed consent will be required prior to randomization.

Patients will be eligible for the study if they have: 1 ) ischemic symptoms such as angina pectoris for more than 20 minutes; 2) occurrence of previous symptoms less than 72 hours before randomization; 3) cardiac troponin levels above the 99th percentile; and 4) culprit lesion containing thrombus (TIMI thrombus grade 2 to 5 within the lesion) and intended early PCI.

Exclusion criteria will be presence of cardiogenic shock, STEMI, no identifiable culprit lesion, coronary morphology ineligible for thrombectomy (for example, very tortuous vessels or severe calcification), indication for acute bypass surgery, age less than 18 or more than 90 years, pregnancy, current participation in another clinical study, co-morbidity with limited life expectancy of less than 6 months or contraindications to CMR at study entry (for example, severe claustrophobia, implanted pacemakers or defibrillators, relevant metallic implants, known allergy to gadolinium, severe renal failure with creatinine clearance $<30 \mathrm{ml} / \mathrm{h}$ ), and contraindications for treatment with heparin, aspirin, or thienopyridines.

\section{Randomization and patient treatment}

Patient randomization will be performed centrally, with a randomization ratio of 1:1 $(n=230$ patients per group; see Figure 1 for study flow) using an internet-based randomization program. No stratification will be performed. All participating hospitals are high-volume tertiary care centers with experienced cardiologists performing all interventions. Manual thrombectomy will be performed in the thrombus aspiration group with an aspiration catheter commonly used in daily clinical routine (Eliminate; ${ }^{\circ}$ Terumo Europe, Leuven, Belgium). In the standard PCI group, patients will be treated by conventional PCI in accordance with local practice without thrombectomy. Cross-over is not planned; however, if this occurs, the reasons will be recorded. Manual thrombectomy will be the only difference between the groups, and all other therapeutic procedures will be similar in both treatment arms.

In vessels with a diameter of greater or equal than 2.5 $\mathrm{mm}$, stent implantation is recommended. In both groups, the decision whether to use bare-metal or drugeluting stents or whether to use glycoprotein IIb/IIIa inhibitors will be left to the discretion of the interventional cardiologist and the treatment decided upon will be recorded. Anticoagulant therapy including administration of heparin, bivalirudin, or low-molecular-weight heparin will be carried out in accordance with standard clinical practice and current guidelines. Additional treatment of other stenoses will be carried out if the interventional cardiologist deems it advisable, and will be recorded. Concomitant drug therapy for the treatment of NSTEMI will be administered based on the decision of the individual investigator and the common practice of the participating hospitals, in accordance with current guidelines. Anti-platelet therapy with initial loading dose will consist of aspirin $100 \mathrm{mg}$ per day in combination with prasugrel $60 \mathrm{mg}$ loading dose and subsequently 10 $\mathrm{mg}$ for 12 months for patients without contraindication to prasugrel. Patients pre-treated with clopidogrel should be switched to prasugrel whereas patients pre-treated with ticagrelor will not be switched. Post-infarction treatment includes administration of angiotensinconverting enzyme inhibitors or angiotensin-II-receptor antagonists, beta-blockers, and statins $[11,12]$.

\section{Cardiac magnetic resonance image acquisition}

The primary endpoint (late $\mathrm{MO}$ ) and secondary endpoints such as early MO, infarct size, and myocardial salvage will be assessed by CMR performed on a 1.5 or 3.0 Tesla scanner. Imaging will be performed on days 1 to 4 after randomization, using a standard scanning protocol (Figure 2). In brief, late MO and infarct size will be assessed in delayed enhancement short-axis images covering the whole ventricle acquired at middiastole approximately 15 minutes after bolus injection (0.2 $\mathrm{mmol} / \mathrm{kg}$ bodyweight) of gadobutrol (Gadovist, Schering, Germany). An inversion recovery (IR) turbo gradient echo sequence will be used for image acquisition. The individual IR pre-pulse delay will be defined to obtain the maximal contrast between viable and necrotic myocardium. Early MO will be assessed 1 to 2 minutes after contrast-medium injection. For determination of edema/area at risk, short-axis slices covering the entire left ventricle (LV) before contrast administration will be obtained using a T2-weighted triple IR turbo spin-echo sequence. Assessment of LV function and volumes will be performed using a standard steady-state free precession technique, acquiring short-axis slices from base to apex and also horizontal and vertical long-axis views. Late and early MO, infarct size and area at risk will be expressed as percentages of the left ventricular mass (\% $L V)$, given by the sum of the mass of late and early MO regions, late enhancement and edema for all slices divided by the sum of the LV myocardial cross-sectional mass. The myocardial salvage index will be calculated as edema/area at risk minus infarct size divided by area at 


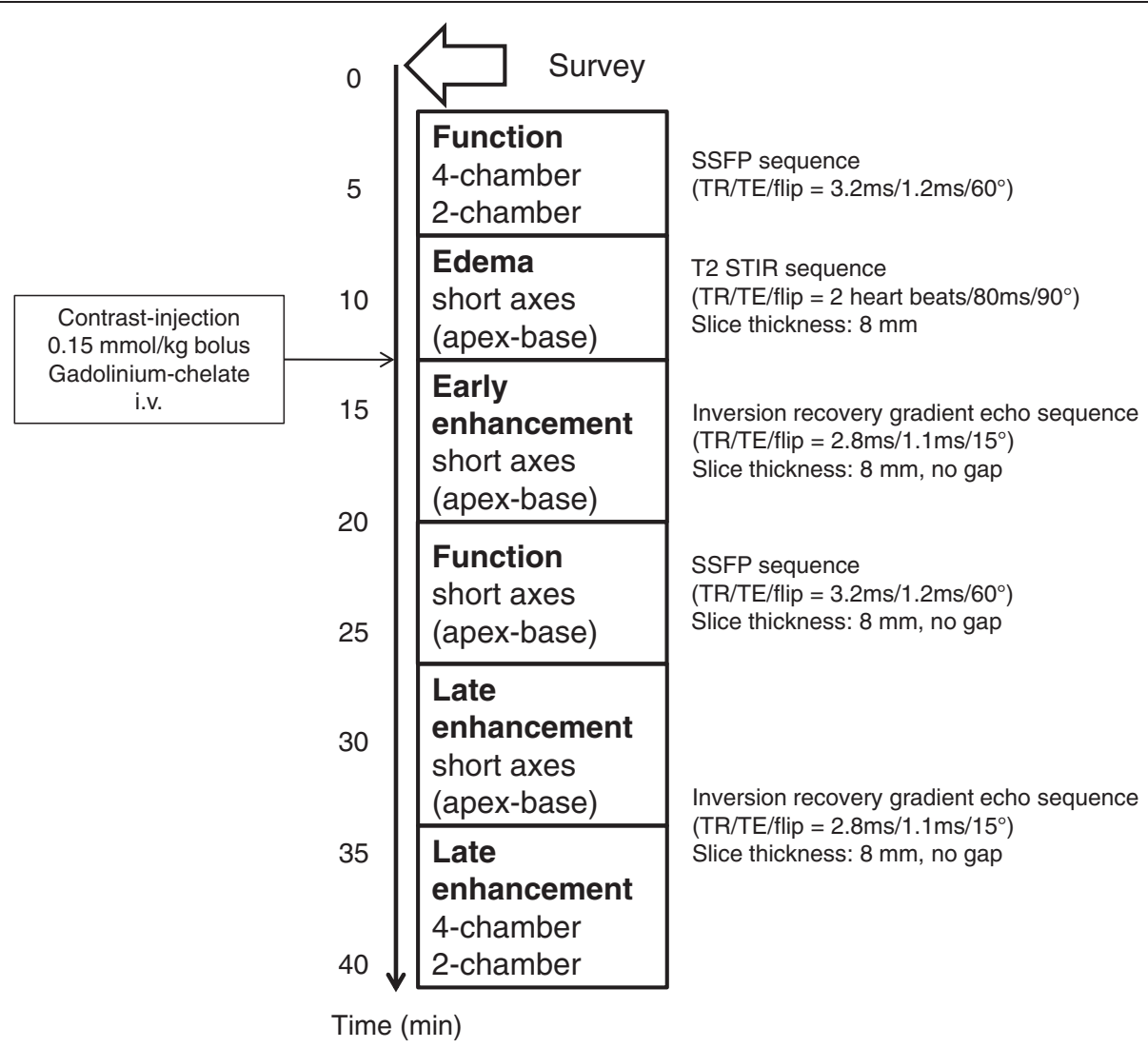

Figure 2 Cardiac magnetic resonance imaging scan protocol. Flip, flip angle; IR, inversion recovery; SSFP, steady-state free precession; STIR, short tau inversion recovery; repetition time; $\mathrm{TE}$, echo time.

risk as described previously [24]. LVEF will be calculated from the short-axis functional views. The scan protocol and image analysis will be standardized at all sites and have been used effectively in previous studies [25-27]. All participating centers are experienced in using this type of conducted scan. CMR images will be sent to the CMR core laboratory at the University of Leipzig - Heart Center, Germany. The CMR core laboratory is highly experienced, has excellent reproducibility and low inter-observer and intra-observer variability for infarct size and myocardial salvage assessment, and has previously served as a core laboratory in studies of acute coronary syndromes [25-27]. Image analysis for the assessment of early and late MO, area at risk and infarct size, and total LV mass and volumes using a semi-automatic approach will be performed in anonymized form by blinded operators.

\section{Assessment of secondary angiographic endpoints}

Both before and after PCI, the culprit vessel should be visualized in several projections in order to obtain particularly precise display of the distal segments. Evaluation of thrombus burden will be performed before and after PCI, in accordance with the TIMI thrombus grade scale.
The flow within the infarct-related artery will be assessed using the TIMI criteria and the corresponding myocardial blush grades [28,29]. For the assessment of myocardial blush, it is especially important for the investigators to provide film material with a sufficiently long duration to allow evaluation of myocardial perfusion. A central blinded analysis for TIMI flow, blush grade, and thrombus burden will be performed at the angiographic core lab of the University of Saarland.

\section{Biomarker sub-study}

In a single-center sub-study (University Leipzig - Heart Center, Germany) blood will be collected from the femoral sheath (peripheral blood) and from the infarctrelated coronary artery for biomarker sub-studies for approximately 200 patients. In this technique, the plasma is separated by centrifugation at $3500 \mathrm{~g}$ for 10 minutes, and aliquots are stored at $-80^{\circ} \mathrm{C}$ until used. Multiple biomarkers such as markers of CHF, inflammation, or myocardial-derived microRNAs (miRNAs) will be analyzed. In addition, assessment of platelet function will be performed. 


\section{Thrombus composition sub-study}

In a two-center study (University of Leipzig - Heart Center and University of Tübingen, both in Germany) that will include approximately 100 patients, the composition and key determinants of aspirated thrombi will be analyzed. This will include histological and immunohistological analysis, and analysis of specific biomarkers such as thrombus miRNAs. The probes will be stored at $-80^{\circ} \mathrm{C}$ and transferred to the core facility at the University of Tübingen.

\section{Data and statistical analysis}

The aim of the study is to demonstrate superiority of manual thrombus aspiration versus standard PCI without thrombus aspiration, based on the primary endpoint of late MO in the study patients.

At the time of planning the study, the only available data on MO in NSTEMI were from a study on a small number $(\mathrm{n}=25)$ of patients [30]. Thus, we initially based our sample-size calculation on previous in-house studies of the University of Leipzig - Heart Center in patients with STEMI and NSTEMI. An absolute difference in the primary endpoint (late $\mathrm{MO}$ ) of $1.0 \% \mathrm{LV}$ between the two treatment groups was expected, and a standard deviation of 3.3\%LV was assumed [17,26,27,31]. Based on $\alpha=5 \%$, a two-sided hypothesis test, and a statistical power of $80 \%, 172$ patients per group would be needed to reject the null hypothesis of no difference between groups.

In the intervening time, two other studies on $\mathrm{MO}$ and NSTEMI were published, which reported a lower prevalence and extent of MO than expected [20,32]. Thus, the Steering Committee decided to perform a preliminary analysis on CMR parameters, including the first 60 patients enrolled in TATORT-NSTEMI. A prevalence of MO of approximately $40 \%$ with a median extent of $1.0 \% \mathrm{LV}$ was oberserved. We thus decided to critically revise our sample-size calculation and adapt it based on the new available data and the preliminary analysis. We now expect an absolute difference in the primary endpoint (late $\mathrm{MO}$ ) of $0.5 \% \mathrm{LV}$ between the two treatment groups with a mean extent of $1.0 \% \mathrm{LV}$ for late $\mathrm{MO}$ in the whole study cohort [20,32]. This difference is judged as clinically relevant $[17,20,26,27,31]$. A standard deviation of $1.8 \% \mathrm{LV}$ is assumed [17,20,26,27,31,32]. Based on $\alpha=$ $5 \%$, a two-sided hypothesis test, and a statistical power of $80 \%, 204$ patients per group are needed to reject the null hypothesis of no difference between groups. On the basis of clinical experience and data in STEMI, it is further expected that it will not be possible to perform thrombectomy for $5 \%$ of all patients in the thrombectomy group, and that $10 \%$ of all patients, either will not undergo a CMR examination or their CMR images will be of insufficient quality for analysis $[17,26,27]$. This will result in a sample size of $2 \times 230$ randomized patients per group. We thus decided to include 60 additional patients after critical revision and adaptation of our initial sample-size calculation. This protocol modification was approved by the central ethics committee at the University of Leipzig. Sample-size calculation was performed using SigmaStat software (Version 3.5; Systat Software Inc., Chicago, IL, USA).

Pre-specified subgroup analyses by gender, presence of diabetes, TIMI thrombus burden (2 to 4 versus 5), TIMI flow pre-PCI ( 0 to 1 versus 2 to 3 ), and time from most recent symptoms to occurrence of PCI $(<6$ versus $\geq 6$ hours), and additional administration of glycoprotein IIb/IIIa inhibitors will be performed.

All patients will be analyzed on an intention-to-treat basis. The differences in primary and secondary endpoints between the treatment groups will be assessed using an unpaired $t$-test. In addition, as a secondary analysis, the differences between the treatment groups in the main outcome will be assessed using an unpaired $t$ test adjusted by baseline values (ANCOVA). Categorical variables will be analyzed by Fisher's exact test. The 95\% confidence interval (CI) for differences in quantitative data will be displayed. For clinical events, relative risks and $95 \%$ CI will be reported. To identify factors associated with the observed results, univariate and multivariate regression analysis will be performed. To assess clinical outcome, Kaplan-Meier curves with log-rank comparison and Cox regression analyses will be performed. A two-tailed $P$-value of $<0.05$ will be considered significant.

\section{Study organization}

The TATORT-NSTEMI Steering Committee is chaired by Prof. Dr. Holger Thiele (University of Leipzig - Heart Center, Leipzig, Germany), co-chaired by Prof. Dr. Uwe Zeymer, (Institut für Herzinfarktforschung [IHF], Ludwigshafen, Germany), and Prof. Dr. Bruno Scheller (University of Saarland, Campus Homburg/Saar, Homburg, Germany). The Steering Committee is responsible for the scientific content of the protocol and oversees the trial operations, and will perform the preparation of the primary manuscript and other publications arising from the TATORT-NSTEMI trial. Each participating center has a coordinator with extensive clinical-trial experience. All trial-related processes will follow the standard operating procedures of the IHF, which is an independent clinical-research organization providing biometry, study coordination, monitoring, and data management. Central monitoring will include a timely query-management process based on consistency and plausibility checks automatically generated from the database, combined with a dunning process for missing documentation and a reminder system in advance for upcoming visits. On- 
site monitoring will include initiation, regular visits, and close-out. During recruitment and follow-up, all centers will be visited regularly to check adherence to good clinical practice and trial protocol. The focus of the visits will be on the verification of informed-consent documents, eligibility criteria, key primary and secondary endpoints, and safety aspects.

The trial will be monitored by an independent DSMB. The CEC will adjudicate all clinical endpoints. The trial follows a standard study organization with a Steering Committee, DSMB, and CEC.

The study has been approved by the central ethical committee at the University of Leipzig and at all local ethics committees at the participating sites.

\section{Discussion}

Despite early invasive strategies and optimal medical treatment, morbidity and mortality rates for NSTEMI and STEMI continue to be high. Thus, further improvement of the initial therapy is necessary to improve patient outcome. The pivotal TAPAS (Thrombus Aspiration during Percutaneous coronary intervention in Acute myocardial infarction Study) trial of patients with STEMI, which compared a strategy with thrombectomy with a strategy without thrombectomy, showed a reduction in cardiac mortality at 1 year [1]. By contrast, other randomized, controlled studies did not observe superiority of thrombectomy over standard PCI with respect to surrogate endpoints of reperfusion success $[33,34]$. However, metaanalyses in patients with STEMI showed a mortality-related benefit after thrombectomy compared with PCI alone $[2,3]$. Therefore, the current guidelines of the ESC on revascularization therapy in patients with STEMI reperfused by primary PCI have increased the recommendation class for manual thrombectomy from the previous class IIb, level of evidence B to class IIa, level of evidence A [4]. In line with the ESC guidelines, thrombectomy is strongly recommended by the AHA/ACC guidelines (class IIa, level of evidence B) [5].

Notably, in the majority of both STEMI and NSTEMI cases, the key pathophysiological substrate is rupture of a vulnerable plaque with subsequent coronary thrombosis. Whereas in STEMI the thrombus is mostly fibrinrich leading to total vessel occlusion, the thrombus in many patients with NSTEMI is predominantly plateletrich and unstable. leading to partial or intermittent occlusion of the coronary vessel [35]. The majority of patients with NSTEMI display relevant thrombus burden and thus might benefit from thrombectomy [6,7]; however, current guidelines do not recommend thrombus aspiration in patients with NSTEMI [11,12]. This is mainly because of the lack of data for patients with NSTEMI, with only one published prospective observational study to date [7]. In that study, which enrolled 70 patients with NSTEMI, a thrombus was detected by the initial angiogram in approximately $50 \%$ of participants. No complications occurred during thrombectomy. Manual thrombus aspiration was associated with a significant reduction of the TIMI thrombus score (score $4 / 5$ in $40 \%$ of patients before thrombectomy versus $7 \%$ after thrombectomy) and an increase in the rate of TIMI flow 3 (in $36 \%$ pre-thrombectomy versus $66 \%$ post-thrombectomy). After thrombus aspiration, direct stenting without predilatation was possible in 39 patients (55.7\%). Thus, this study showed that thrombus aspiration in patients with NSTEMI is feasible, effective, and safe.

However, RCTs are necessary to investigate whether thrombus aspiration in NSTEMI results in improved angiographic, functional, and clinical outcomes compared with conventional PCI. Currently, one additional randomized single-center study comparing thrombectomy with PCI alone is ongoing (TAPAS-II study) [36]. The primary endpoint of that study is myocardial blush grade, and the study aims to enroll 560 patients with NSTEMI. The main limitations of that study are its single-center design and the performance of unselected thrombectomy in all patients, independent of thrombus presence. In addition, the primary endpoint of myocardial blush grade is only an indirect marker for myocardial perfusion. By contrast, the primary endpoint of our proposed study, late MO by CMR, allows direct visualization and quantification of no-reflow and microcirculatory impairment $[15,16]$. Both myocardial blush and MO are surrogate endpoints, and evidently assessing clinical outcome as primary endpoint would lead to more robust data. However, late $\mathrm{MO}$ has been shown to be a strong independent predictor for clinical outcome, including mortality, after acute coronary syndromes [17-19]. The main pathophysiological mechanism for the development of $\mathrm{MO}$ is distal embolization [13,14]. Because manual thrombus removal reduces thrombus burden and thereby distal embolization, the primary endpoint of the current trial (MO) and the studied intervention (manual thrombectomy) are directly and causally linked with each other on a pathophysiological basis.

Previous data for patients with STEMI indicate that in approximately $10 \%$ of all patients the thrombus aspiration catheter fails to cross the lesion [37]. Because lesions in NSTEMI are often less complex and frequently display lower-grade stenoses, it can be expected that it will not be possible to perform thrombectomy in a smaller percentage (approximately $5 \%$ ) of all patients in the thrombectomy group. To reduce the rate of unsuccessful thrombectomy, presence of a coronary morphology ineligible for thrombectomy (for example, patients with very tortuous vessels or severe calcification) will be a primary exclusion criterion. In addition, only interventional cardiologists experienced in manual thrombectomy 
will participate in this trial. Finally, blinding will not be possible as a result of the intervention used. However, several methods to restrict bias will be implemented, such as a central computerized randomization system; a blinded CEC to assess all relevant clinical events; a blinded CMR and angiographic core laboratory; and high standard requirements concerning the clinical level and experience of centers and investigators in terms of numbers of PCIs performed, cases of NSTEMI treated, and participation in cardiologic clinical trials.

In clinical trials using CMR parameters as surrogate endpoints, the time period for CMR image acquisition after the index event or randomization is of importance for the final quality and reproducibility of the results. However, previous studies analyzing the influence of timing of CMR image acquisition on CMR parameters led to inconsistent results. In contrast to previously published animal data with stable infarct size and MO measurements between day 2 and 7 after the index event [38],there has been increasing evidence recently based on findings in humans that infarct size decreases within the first week after the initial event [39-42]. However, these studies included only small study sample sizes, and to date, the precise devolution of the infarcted areal within the first days has not been investigated. Thus, based on current data and practicability of performance of CMR within clinical routine times to assure high-quality image acquisition, we chose to acquire CMR images within the narrow time period of 1 to 4 days after randomization. Furthermore, we do not expect differences in timing of CMR in both treatment groups. Thus, even if there were to be an increase or decrease in MO over time in humans, this would not affect the study results.

\section{Trial status}

Recruitment began in March 2011 and of December 2012, almost 360 patients had been enrolled. Given these inclusion rates, completion of enrollment is expected by July 2013. It is expected that complete data will be available in September 2013.

\footnotetext{
Abbreviations

AHA/ACC: American Heart Association/American College of Cardiology; CEC: Clinical Endpoints Committee; CHF: congestive heart failure; $\mathrm{Cl}$ : Confidence interval; CMR: Cardiac magnetic resonance; DSMB: Data Safety Monitoring Board; ESC: European Society of Cardiology; IHF: Institut für Herzinfarktforschung; LV: Left ventricle; LVEF: Left ventricular ejection fraction; MO: Microvascular obstruction; NSTEMI: Non-ST-elevation myocardial infarction; PCl: Percutaneous coronary intervention; RCT: Randomized controlled trial (RCT); STEMI: ST-elevation myocardial infarction; TATORTNSTEMI: Thrombus Aspiration in ThrOmbus containing culpRiT lesions in Non-ST-Elevation Myocardial Infarction; TIMI: Thrombolysis in myocardial infarction.
}

\section{Competing interests}

The authors do not have competing interests concerning the TATORTNSTEMI trial.

\section{Authors' contributions}

All authors have made substantial contributions to conception and design of the current trial and have been involved in drafting the manuscript or revising it critically for important intellectual content. All authors have given final approval of the manuscript.

\section{Acknowledgments}

The TATORT-NSTEMI trial is an investigator-initiated study which is supported by unrestricted grants from Terumo Europe, Leuven, Belgium and Lilly Germany, Bad Homburg, Germany. The authors are solely responsible for the design and conduct of the study and all study analyses, the drafting and editing of the paper and its final contents. The aforementioned companies have no influence on the study design, data collection, data analysis, and final drafting of this manuscript.

Finally, we acknowledge the support of the members of the DSMB including Kurt Huber, Wilhelminenspital der Stadt Wien, Vienna, Austria, Hans-Richard Arntz, Charité, Campus Benjamin Franklin, Berlin, Germany and Karl Wegscheider, University Hamburg-Eppendorf, Hamburg, Germany.

\section{Author details}

${ }^{1}$ Department of Internal Medicine/Cardiology, University of Leipzig - Heart Center, Struempellstr. 39, Leipzig 04289, Germany. ${ }^{2}$ Department of Internal Medicine III, University of Saarland, Kirrberger Str. 100, Homburg 06841, Germany. ${ }^{3}$ Department of Cardiology, Zentralklinik Bad Berka,

Robert-Koch-Allee 9, Bad Berka 99437, Germany. ${ }^{4}$ Department of Cardiology/ Cardiovascular Medicine, University of Tuebingen, Otfried-Mueller-Str. 10, Tuebingen 72076, Germany. ${ }^{5}$ Department of Internal Medicine II, Klinikum Frankfurt/Oder, Muellroser Chaussee 7, Frankfurt/Oder 15236, Germany. ${ }^{6}$ Department of Internal Medicine, Unfallkrankenhaus Berlin, Warener Str. 7, Berlin 12683, Germany. ${ }^{7}$ Department of Internal Medicine I, University of Leipzig, Liebigstr. 20, Leipzig 04103, Germany. ${ }^{8}$ Institut für

Herzinfarktforschung, Klinikum der Stadt Ludwigshafen, Bremserstr. 79 Ludwigshafen 67063, Germany.

Received: 24 October 2012 Accepted: 22 March 2013

Published: 25 April 2013

\section{References}

1. Vlaar PJ, Svilaas T, van der Horst IC, Diercks GF, Fokkema ML, de Smet BJ, van den Heuvel AF, Anthonio RL, Jessurun GA, Tan ES, et al: Cardiac death and reinfarction after 1 year in the Thrombus Aspiration during Percutaneous coronary intervention in Acute myocardial infarction Study (TAPAS): a 1-year follow-up study. Lancet 2008, 371:1915-1920.

2. Burzotta F, De Vita M, Gu YL, Isshiki T, Lefevre T, Kaltoft A, Dudek D, Sardella G, Orrego PS, Antoniucci D, et al: Clinical impact of thrombectomy in acute ST-elevation myocardial infarction: an individual patient-data pooled analysis of 11 trials. Eur Heart J 2009, 30:2193-2203.

3. De Luca G, Dudek D, Sardella G, Marino P, Chevalier B, Zijlstra F: Adjunctive manual thrombectomy improves myocardial perfusion and mortality in patients undergoing primary percutaneous coronary intervention for ST-elevation myocardial infarction: a meta-analysis of randomized trials. Eur Heart J 2008, 29:3002-3010.

4. Wijns W, Kolh P, Danchin N, Di Mario C, Falk V, Folliguet T, Garg S, Huber K, James S, Knuuti J, et al: Guidelines on myocardial revascularization. Eur Heart J 2010, 31:2501-2555

5. Kushner FG, Hand M, Smith SC Jr, King SB 3rd, Anderson JL, Antman EM, Bailey SR, Bates ER, Blankenship JC, Casey DE Jr, et al: Focused Updates: ACC/AHA Guidelines for the Management of Patients With ST-Elevation Myocardial Infarction (updating the 2004 Guideline and 2007 Focused Update) and ACC/AHA/SCAI Guidelines on Percutaneous Coronary Intervention (updating the 2005 Guideline and 2007 Focused Update): a report of the American College of Cardiology Foundation/American Heart Association Task Force on Practice Guidelines. Circulation 2009, 2009(120):2271-2306.

6. Bahrmann P, Rach J, Desch S, Schuler GC, Thiele H: Incidence and distribution of occluded culprit arteries and impact of coronary collaterals on outcome in patients with non-ST-segment elevation myocardial infarction and early invasive treatment strategy. Clin Res Cardiol 2011, 100:457-467.

7. Vlaar PJ, Diercks GF, Svilaas T, Vogelzang M, de Smet BJ, van den Heuvel AF, Anthonio RL, Jessurun GA, Tan ES, Suurmeijer AJ, Zijlstra F: The feasibility 
and safety of routine thrombus aspiration in patients with non-ST-elevation myocardial infarction. Catheter Cardiovasc Interv 2008, 72:937-942.

8. Hong YJ, Jeong MH, Choi YH, Ko JS, Lee MG, Kang WY, Lee SE, Kim SH, Park $\mathrm{KH}$, Sim DS, et al: Impact of plaque components on no-reflow phenomenon after stent deployment in patients with acute coronary syndrome: a virtual histology-intravascular ultrasound analysis. Eur Heart J 2011, 32:2059-2066.

9. McEntegart MB, Kirtane AJ, Cristea E, Brener S, Mehran R, Fahy M, Moses JW, Stone GW: Intraprocedural thrombotic events during percutaneous coronary intervention in patients with non-ST-segment elevation acute coronary syndromes are associated with adverse outcomes: analysis from the ACUITY (Acute Catheterization and Urgent Intervention Triage Strategy) trial. J Am Coll Cardiol 2012, 59:1745-1751.

10. Zhao XQ, Theroux P, Snapinn SM, Sax FL: Intracoronary thrombus and platelet glycoprotein Ilb/Illa receptor blockade with tirofiban in unstable angina or non-Q-wave myocardial infarction. Angiographic results from the PRISM-PLUS trial (Platelet receptor inhibition for ischemic syndrome management in patients limited by unstable signs and symptoms). PRISM-PLUS Investigators. Circulation 1999, 100:1609-1615.

11. Hamm CW, Bassand JP, Agewall S, Bax J, Boersma E, Bueno H, Caso P, Dudek D, Gielen S, Huber K, et al: ESC Guidelines for the management of acute coronary syndromes in patients presenting without persistent ST-segment elevation: the task force for the management of acute coronary syndromes (ACS) in patients presenting without persistent ST-segment elevation of the European Society of Cardiology (ESC). Eur Heart J 2011, 32:2999-3054

12. Wright RS, Anderson JL, Adams CD, Bridges CR, Casey DE Jr, Ettinger SM, Fesmire FM, Ganiats TG, Jneid H, Lincoff AM, et al: ACCF/AHA Focused Update of the Guidelines for the Management of Patients With Unstable Angina/ Non-ST-Elevation Myocardial Infarction (Updating the 2007 Guideline): a report of the American College of Cardiology Foundation/ American Heart Association Task Force on Practice Guidelines. Circulation 2011, 2011(123):2022-2060.

13. Jaffe $R$, Charron T, Puley G, Dick A, Strauss BH: Microvascular obstruction and the no-reflow phenomenon after percutaneous coronary intervention. Circulation 2008, 117:3152-3156.

14. Niccoli G, Burzotta F, Galiuto L, Crea F: Myocardial no-reflow in humans. J Am Coll Cardiol 2009, 54:281-292.

15. Nijveldt R, Beek AM, Hofman MB, Umans VA, Algra PR, Spreeuwenberg MD, Visser CA, van Rossum AC: Late gadolinium-enhanced cardiovascular magnetic resonance evaluation of infarct size and microvascular obstruction in optimally treated patients after acute myocardial infarction. J Cardiovasc Magn Reson 2007, 9:765-770.

16. Orn S, Manhenke C, Greve OJ, Larsen Al, Bonarjee W, Edvardsen T, Dickstein K: Microvascular obstruction is a major determinant of infarct healing and subsequent left ventricular remodelling following primary percutaneous coronary intervention. Eur Heart J 2009, 30:1978-1985.

17. de Waha S, Desch S, Eitel I, Fuernau G, Zachrau J, Leuschner A, Zachrau J, Schuler $\mathrm{G}$, Thiele $\mathrm{H}$ : Impact of early versus late microvascular obstruction assessed by magnetic resonance imaging on long-term outcome after st-elevation myocardial infarction - a comparison to traditional prognostic markers. Eur Heart J 2010, 31:2660-2668.

18. Larose $E$, Rodes-Cabau J, Pibarot P, Rinfret $S$, Proulx G, Nguyen CM, Dery JP Gleeton $\mathrm{O}$, Roy L, Noel B, et al: Predicting late myocardial recovery and outcomes in the early hours of ST-segment elevation myocardial infarction traditional measures compared with microvascular obstruction, salvaged myocardium, and necrosis characteristics by cardiovascular magnetic resonance. J Am Coll Cardiol 2010, 55:2459-2469.

19. Wu KC, Zerhouni EA, Judd RM, Lugo-Olivieri CH, Barouch LA, Schulman SP, Blumenthal RS, Lima JAC: Prognostic significance of microvascular obstruction by magnetic resonance imaging in patients with acute myocardial infarction. Circulation 1998, 97:765-772.

20. Cochet A, Lalande A, Lorgis L, Zeller M, Beer JC, Walker PM, Touzery C, Wolf $J E$, Cottin Y, Brunotte F: Prognostic value of microvascular damage determined by cardiac magnetic resonance in non ST-segment elevation myocardial infarction: comparison between first-pass and late gadolinium-enhanced images. Invest Radiol 2011, 45:725-732.

21. Raman SV, Simonetti OP, Winner MW 3rd, Dickerson JA, He X, Mazzaferri EL $J$ r, Ambrosio G: Cardiac magnetic resonance with edema imaging identifies myocardium at risk and predicts worse outcome in patients with non-ST-segment elevation acute coronary syndrome. J Am Coll Cardiol 2010, 55:2480-2488.

22. Thygesen K, Alpert JS, Jaffe AS, Simoons ML, Chaitman BR, White HD, Katus HA, Apple FS, Lindahl B, Morrow DA, et al: Third universal definition of myocardial infarction. Eur Heart J 2012, 33:2551-2567.

23. The GUSTO investigators: An international randomized trial comparing four thrombolytic strategies for acute myocardial infarction. N Engl J Med 1993, 329:673-682.

24. Friedrich MG, Abdel-Aty H, Taylor A, Schulz-Menger J, Messroghli D, Dietz R: The salvaged area at risk in reperfused acute myocardial infarction as visualized by cardiovascular magnetic resonance. J Am Coll Cardiol 2008, 51:1581-1587.

25. Patel MR, Smalling RW, Thiele H, Barnhart HX, Zhou Y, Chandra P, Chew D, Cohen M, French J, Perera D, Ohman EM: Intra-aortic balloon counterpulsation and infarct size in patients with acute anterior myocardial infarction without shock: the CRISP AMI randomized trial. JAMA 2011, 306:1329-1337.

26. Thiele H, Eitel I, Meinberg C, Desch S, Leuschner A, Pfeiffer D, Hartmann A, Lotze U, Strauss W, Schuler G: Randomized comparison of pre-hospital -initiated facilitated percutaneous coronary intervention versus primary percutaneous coronary intervention in acute myocardial infarction very early after symptom onset: the LIPSIA-STEMI trial (Leipzig immediate prehospital facilitated angioplasty in ST-segment myocardial infarction). JACC Cardiovasc Interv 2011, 4:605-614.

27. Thiele H, Schindler K, Friedenberger J, Eitel I, Fürnau G, Grebe E, Erbs S, Linke A, Möbius-Winkler S, Kivelitz D, Schuler G: Intracoronary compared with intravenous bolus abciximab application in patients with STelevation myocardial infarction undergoing primary percutaneous coronary intervention. Circulation 2008, 118:49-57.

28. Cannon CP, Battler A, Brindis RG, Cox JL, Ellis SG, Every NR, Flaherty JT, Harrington RA, Krumholz HM, Simoons ML, et al: American College of Cardiology key data elements and definitions for measuring the clinical management and outcomes of patients with acute coronary syndromes. A report of the American College of Cardiology Task Force on Clinical Data Standards (Acute Coronary Syndromes Writing Committee). J Am Coll Cardiol 2001, 38:2114-2130.

29. van 't Hof AW, Liem A, Suryapranata H, Hoorntje JC, de Boer MJ, Zijlstra F: Angiographic assessment of myocardial reperfusion in patients treated with primary angioplasty for acute myocardial infarction: myocardial blush grade. Zwolle Myocardial Infarction Study Group. Circulation 1998, 97:2302-2306.

30. Mewton N, Bonnefoy E, Revel D, Ovize M, Kirkorian G, Croisille P: Presence and extent of cardiac magnetic resonance microvascular obstruction in reperfused non-ST-elevated myocardial infarction and correlation with infarct size and myocardial enzyme release. Cardiology 2009, 113:50-58.

31. Sardella G, Mancone M, Bucciarelli-Ducci C, Agati L, Scardala R, Carbone I, Francone M, Di Roma A, Benedetti G, Conti G, Fedele F: Thrombus aspiration during primary percutaneous coronary intervention improves myocardial reperfusion and reduces infarct size: the EXPIRA (thrombectomy with export catheter in infarct-related artery during primary percutaneous coronary intervention) prospective, randomized trial. J Am Coll Cardiol 2009, 53:309-315.

32. Xu J, Song YB, Hahn JY, Chang SA, Lee SC, Choe YH, Choi SH, Choi JH, Lee $\mathrm{SH}$, Oh JK, Gwon HC: Comparison of magnetic resonance imaging findings in non-ST-segment elevation versus ST-segment elevation myocardial infarction patients undergoing early invasive intervention. Int J Cardiovasc Imaging 2012, 28:1487-1497.

33. Stone GW, Maehara A, Witzenbichler B, Godlewski J, Parise H, Dambrink JH, Ochala A, Carlton TW, Cristea E, Wolff SD, et al: Intracoronary abciximab and aspiration thrombectomy in patients with large anterior myocardial infarction: the INFUSE-AMI randomized trial. JAMA 2012, 307:1817-1826.

34. Lipiecki J, Monzy S, Durel N, Cachin F, Chabrot P, Muliez A, Morand D, Maublant J, Ponsonnaille J: Effect of thrombus aspiration on infarct size and left ventricular function in high-risk patients with acute myocardial infarction treated by percutaneous coronary intervention. Results of a prospective controlled pilot study. Am Heart J 2009, 157:e581-e587.

35. Silvain J, Collet JP, Nagaswami C, Beygui F, Edmondson KE, BellemainAppaix A, Cayla G, Pena A, Brugier D, Barthelemy O, et al: Composition of coronary thrombus in acute myocardial infarction. J Am Coll Cardiol 2011, 57:1359-1367. 
36. Kampinga MA, Vlaar PJ, Fokkema M, Gu YL, Zijlstra F: Thrombus Aspiration during Percutaneous coronary intervention in Acute non-ST-elevation myocardial infarction Study (TAPAS II)-Study design. Neth Heart J 2009, 17:409-413.

37. Vink MA, Kramer MC, Li X, Damman P, Rittersma SZ, Koch KT, van der Wal AC, Tijssen JG, de Winter RJ: Clinical and angiographic predictors and prognostic value of failed thrombus aspiration in primary percutaneous coronary intervention. JACC Cardiovasc Interv 2011, 4:634-642.

38. Wu KC, Kim RJ, Bluemke DA, Rochitte CE, Zerhouni EA, Becker LC, Lima JAC: Quantification and time course of microvascular obstruction by contrastenhanced echocardoigraphy and magnetic resonance imaging following acute myocardial infarction and reperfusion. J Am Coll Cardiol 1998, 32:1756-1764.

39. Dall'Armellina E, Karia N, Lindsay AC, Karamitsos TD, Ferreira V, Robson MD, Kellman P, Francis JM, Forfar C, Prendergast BD, et al: Dynamic changes of edema and late gadolinium enhancement after acute myocardial infarction and their relationship to functional recovery and salvage index. Circ Cardiovasc Imaging 2011, 4:228-236.

40. Engblom H, Hedstrom E, Heiberg E, Wagner GS, Pahlm O, Arheden H: Rapid initial reduction of hyperenhanced myocardium after reperfused first myocardial infarction suggests recovery of the peri-infarction zone: oneyear follow-up by MRI. Circ Cardiovasc Imaging 2009, 2:47-55.

41. Ibrahim T, Hackl T, Nekolla SG, Breuer M, Feldmair M, Schomig A, Schwaiger M: Acute myocardial infarction: serial cardiac MR imaging shows a decrease in delayed enhancement of the myocardium during the 1st week after reperfusion. Radiology 2011, 254:88-97.

42. Mather AN, Fairbairn TA, Artis NJ, Greenwood JP, Plein S: Timing of cardiovascular MR imaging after acute myocardial infarction: effect on estimates of infarct characteristics and prediction of late ventricular remodeling. Radiology 2011, 261:116-126.

doi:10.1186/1745-6215-14-110

Cite this article as: de Waha et al:: Thrombus Aspiration in ThrOmbus containing culpRiT lesions in Non-ST-Elevation Myocardial Infarction (TATORT-NSTEMI): study protocol for a randomized controlled trial. Trials 2013 14:110.

\section{Submit your next manuscript to BioMed Central and take full advantage of:}

- Convenient online submission

- Thorough peer review

- No space constraints or color figure charges

- Immediate publication on acceptance

- Inclusion in PubMed, CAS, Scopus and Google Scholar

- Research which is freely available for redistribution 